\title{
Synthesis, Characterization and Corrosion Inhibition Performance of the Thiourea-chitosan in Acidic Medium
}

\author{
Meng Wang ${ }^{1}$, Jing Zhang ${ }^{1,2, *}$, Qinghai Wang ${ }^{1}$, Min Du $^{1,2}$ \\ ${ }^{1}$ College of Chemistry and Chemical Engineering, Ocean University of China, 266100, Qingdao, \\ China \\ ${ }^{2}$ Key Laboratory of Marine Chemistry Theory and Technology, Ministry of Education, 266100, \\ Qingdao, China \\ *E-mail: dmh217@ouc.edu.cn
}

doi: $10.20964 / 2019.09 .24$

Received: 23 March 2019/ Accepted: 4 May 2019 / Published: 31 July 2019

The water-soluble and green thiourea-chitosan (TUCTS) was synthesized and characterized by the Fourier transform infrared spectroscopy (FT-IR) and elemental analysis method. The corrosion inhibition performance and behavior of TUCTS for Q235 steel in $1 \mathrm{~mol} \cdot \mathrm{L}^{-1} \mathrm{HCl}$ were investigated by weight loss method, surface morphology analysis, potentiodynamic polarization curves and electrochemical impedance spectroscopy (EIS). It is found that the synthesized TUCTS is an excellent corrosion inhibitor and the inhibition efficiency exceeds $90 \%$ at $25^{\circ} \mathrm{C}$. The inhibitor acts as a mixed type inhibitor by geometrical blanketing effect. Thermodynamics analysis demonstrates that the adsorption of TUCTS on Q235 steel surface fit in with the Langmuir model. The adsorption type is both physical and chemical adsorption but chemisorption dominates.

Keywords: Thiourea-chitosan, corrosion inhibitor, acid corrosion

\section{FULL TEXT}

(C) 2019 The Authors. Published by ESG (www.electrochemsci.org). This article is an open access article distributed under the terms and conditions of the Creative Commons Attribution license (http://creativecommons.org/licenses/by/4.0/). 